\title{
A reduced-domain method of structural damage identification: Application to a spectral element beam model
}

\author{
Usik Lee \\ Department of Mechanical Engineering, Inha University 253 Yonghyun-Dong, Nam-Ku, Incheon 402-751, South \\ Korea \\ Tel.: +82 32860 7318; Fax: +82 32866 1434; E-mail: ulee@inha.ac.kr
}

\begin{abstract}
Though there have been many efforts to make the inverse problem of damage identification small by reducing its finite element degrees-of-freedom, there have been few efforts to make it small by reducing its spatial domain of problem. Thus, as the extension of the author's previous work in which the damage identification algorithm was formulated from the dynamic stiffness equation of motion, the present study introduces a spectral element model (SEM)-based reduced-domain method (RDM) of damage identification. In the present RDM, a three-steps process is used to reduce the domain of problem by iteratively searching out and removing damage-free parts of structure in the course of the damage identification analysis. To validate the present RDM, numerically simulated damage identification tests are conducted. The experimental tests for a damaged cantilevered beam specimen show that the present RDM can fairly well locates and quantifies all local damages (i.e., slots) placed along the beam specimen.
\end{abstract}

Keywords: Structural damage, damage identification, reduced-domain method, beam, spectral element model, frequency response function

\section{Introduction}

Since the first attempts by Thompson [1] and Kirmsher [2] to quantify the local structural damages, the structural damage identification (i.e., detection, location and quantification) has been an active research area. The structural damages generated within a structure may lead to the changes in the dynamic characteristics of the structure such as the vibration response, natural frequency, mode shape and the modal damping. Thus, the changes in dynamic characteristics of a structure in turn can be used to identify the structural damages within the structure. In the literature, there have been appeared a variety of structural damage identification methods (SDIM) and the extensive reviews on the subject can be found in the references [36]. An intensive literature survey shows that the fi- nite element models have been widely used in most structure-model based-SDIM including the finite element model (FEM)-update methods (e.g. [7-9]) and the experimental-data-based methods (e.g. [10-25]).

In FEM-update methods, it is assumed that an FEM of a structure is available and the parameters of the stiffness and mass matrices can be updated to match with the experimental modal analysis model. The parameter changes of the stiffness and mass matrices between the intact state and damaged state are the used to detect, locate and quantify the structural damage. However, because the degrees-of-freedom (DOF) of FEM are in general much larger than those of the experimental modal analysis model, it is inevitable to reduce the DOF of FEM. Reducing the DOF of FEM may results in the loss of physical interpretability along with the stiffness diffusion phenomena that may provide fault damage 
identification. Thus, as the alternatives to the FEMupdate methods, the experimental-data-based methods have been proposed in the literature.

The experimental-data-based SDIM derived from FEM eigenvalue problems can be classified into several groups depending on what experimental data are used to detect, locate, and quantify structural damages. They include the natural frequencies [10-14], mode shapes or mode shape curvatures $[15,16]$, transfer function parameters [17], flexibility matrix [18], residual forces $[19,20]$, substructural flexibilities [21] and FRF data [22-25]. It has been well recognized that the FRFdata offer certain advantages over modal data, because the modal extraction process is not required. Furthermore, the FRF data may provide more information on the structural damage by including the higher vibration modes that are not in frequency range of measurement.

For the reliable damage identification, a sufficiently accurate structure-model should be used. However, as a drawback of FEM, it has been well recognized that very fine meshes should be used to obtain satisfactory high frequency dynamic responses. This will increase the DOF to render the FEM-based SDIM not practical for real time damage identification. Thus, to improve the accuracy of structure model and also to make use of the inherent advantages of FRF-data over the modal data, Park et al. [24] used the spectral element bar model and FRF-data to locate the damages within a free-free bar, and Lee and Shin [25] proposed a spectral element model (SEM)-based SDIM in a general form, in which the measured FRF-data can be used, and then applied it to a beam structure. Because the spectral element matrices (which are often called the exact dynamic stiffness matrices) formulated from the exact dynamic (i.e., frequency-dependent) shape functions are used in SEM, only one finite element is enough for a uniform structure element, regardless of its dimensions, to obtain exact dynamic characteristics of the structure element [26,27]. Accordingly this may reduce drastically the number of DOF to be manipulated in the damage identification analysis.

Because the structural damage identification is the inverse problem, it is desirable to reduce the DOF of FEM for easy and efficient manipulation of the finite element models, without significantly degrading the damage identification results. In the literature, various techniques have been used to reduce the DOF of FEM. They include the Guyan reduction technique [27,28], the dynamic condensation technique [29], and the improved reduced system (IRS) [30]. As the severe structural damages are localized at a few small areas in fact, rather than spread wide over whole structure, one doesn't need to examine whole (spatial) domain of structure for damage. Thus, in addition to the efforts to reduce the DOF of FEM or as the alternatives to the efforts, it is desirable to reduce the domain of structure to be examined by removing the parts of structure where the probability of damage is extremely low.

Thus, the purposes of the present study are to apply a concept of reduced-domain method (RDM) to the SEM-based damage identification theory that was derived in the author's previous work [25], and then to verify the reduced-domain method experimentally as well as numerically, by applying it to a beam structure.

\section{Development of reduced-domain method}

\subsection{Damage identification algorithm}

By following the well-defined spectral element formulation procedure [26,27], the forced vibration of a structure system can be represented by

$$
\begin{aligned}
& {[\mathbf{S}(\omega)]\{\mathbf{U}(\omega)\}=\{\mathbf{P}(\omega)\} \text { for intact state }} \\
& {[\overline{\mathbf{S}}(\omega)]\{\overline{\mathbf{U}}(\omega)\}=\{\mathbf{P}(\omega)\} \text { for damaged state }}
\end{aligned}
$$

where $\omega$ is the circular frequency. The frequencydependent matrix $[\mathbf{S}]$ represents the spectral system matrix (or the exact dynamic stiffness matrix) of the structure system. The vectors $\{\mathbf{U}\}$ and $\{\mathbf{P}\}$ represent the spectral components of nodal degrees-of-freedom vector (simply, nodal DOF) and those of externally applied nodal forces vector (simply, nodal forces), respectively. The quantities with over-bar (-) represent the quantities in the damaged state.

The matrix $[\mathbf{S}]$ is considered as the known quantity because it will be so determined that Eq. (1a) represents the refined structure model for the intact structure. The vector $\{\overline{\mathbf{U}}\}$ is also considered as the known quantity because it will be measured directly from the damaged structure. However, the matrix $[\overline{\mathbf{S}}]$ is the unknown quantity because it should depend on the not-yet-known current damage state. The matrix $[\overline{\mathbf{S}}]$ can be represented by

$$
[\overline{\mathbf{S}}(\omega)]=[\mathbf{S}(\omega)]+[\Delta \mathbf{S}(\omega)]
$$

where $[\Delta \mathbf{S}]$ is the perturbed spectral system matrix generated by the presence of damage.

Applying Eq. (2) into Eq. (1) gives

$$
\{\mathbf{P}(\omega)\}-[\mathbf{S}(\omega)]\{\overline{\mathbf{U}}(\omega)\}=[\Delta \mathbf{S}(\omega))]\{\overline{\mathbf{U}}(\omega)\}(3)
$$


Now express the matrices and vectors in Eq. (3) in the partitioned forms as

$$
\begin{aligned}
& \{\mathbf{P}(\omega)\}=\left\{\begin{array}{l}
\mathbf{P}_{m}(\omega) \\
\mathbf{P}_{s}(\omega)
\end{array}\right\}=\left\{\begin{array}{c}
\mathbf{F}(\omega) \\
\mathbf{0}
\end{array}\right\}, \\
& \{\overline{\mathbf{U}}(\omega)\}=\left\{\begin{array}{l}
\overline{\mathbf{U}}_{m}(\omega) \\
\overline{\mathbf{U}}_{s}(\omega)
\end{array}\right\} \\
& {[\mathbf{S}(\omega)]=\left[\begin{array}{l}
\mathbf{S}_{m m}(\omega) \mathbf{S}_{m s}(\omega) \\
\mathbf{S}_{s m}(\omega)
\end{array}\right],} \\
& {[\overline{\mathbf{S}}(\omega)]=\left[\begin{array}{l}
\overline{\mathbf{S}}_{m m}(\omega) \\
\bar{S}_{s m}(\omega) \overline{\mathbf{S}}_{m s}(\omega)
\end{array}\right]} \\
& {[\Delta \mathbf{S}(\omega)]=\left[\begin{array}{ll}
\overline{\mathbf{S}}_{m m}-\mathbf{S}_{m m} & \overline{\mathbf{S}}_{m s}-\mathbf{S}_{m s} \\
\overline{\mathbf{S}}_{s m}-\mathbf{S}_{s m} & \overline{\mathbf{S}}_{s s}-\mathbf{S}_{s s}
\end{array}\right]}
\end{aligned}
$$

where the subscripts $m$ and $s$ denote the quantities corresponding to the 'master' nodal DOF vector $\left\{\overline{\mathbf{U}}_{m}\right\}$ and the 'slave' nodal DOF vector $\left\{\overline{\mathbf{U}}_{s}\right\}$, respectively, and $\{\mathbf{0}\}$ denotes the zero vector.

Applying Eq. (4) into Eq. (3) and conducting a lengthy manipulation yields

$$
\{\mathbf{F}(\omega)\}-[\mathbf{X}(\omega)]\left\{\overline{\mathbf{U}}_{m}(\omega)\right\}[\mathbf{Y}(\omega)]\left\{\overline{\mathbf{U}}_{m}(\omega)\right\}
$$

with

$$
\begin{aligned}
& {[\mathbf{X}(\omega)]=\left[\mathbf{S}_{m m}\right]-\left[\mathbf{S}_{m s}\right]\left[\mathbf{S}_{s s}\right]^{-1}\left[\mathbf{S}_{s m}\right]} \\
& {[\mathbf{Y}(\omega)]=[\mathbf{T}]^{T}[\Delta S][\mathbf{T}]} \\
& {[\mathbf{T}(\omega)]=\left[\begin{array}{c}
{[\mathbf{I}]} \\
-\left[\mathbf{S}_{s s}\right]^{-1}\left[\mathbf{S}_{s m}\right]
\end{array}\right]}
\end{aligned}
$$

where $[\mathbf{I}]$ denotes the identity matrix. By representing the master nodal DOF vector $\left\{\overline{\mathbf{U}}_{m}\right\}$ in Eq. (5) in terms of the inertance FRF vector $\left\{\mathbf{A}_{m}\right\}$, Eq. (5) can be rewritten as

$$
\begin{array}{r}
\{\boldsymbol{\delta}\}+\frac{1}{\omega^{2}}[\mathbf{X}(\omega)]\left\{\mathbf{A}_{m}(\omega)\right\}=-\frac{1}{\omega^{2}}[\mathbf{Y}(\omega)] \\
\left\{\mathbf{A}_{m}(\omega)\right\}
\end{array}
$$

where $\{\boldsymbol{\delta}\}$ is the nodal forces locator vector having a unit value only at the component corresponding to the node at which a harmonic point force is applied, and the components of the inertance FRF vector $\left\{\mathbf{A}_{m}\right\}$ are defined by the ratio of the acceleration to the applied force as [32]

$$
\begin{aligned}
A_{m i} & \equiv-\omega^{2} \frac{\bar{U}_{m i}}{F_{i}}(i=1,2, \ldots, M) \text { or } \\
\left\{\mathbf{A}_{m}\right\} & \equiv-\omega^{2}\left\{\frac{\overline{\mathbf{U}}_{m}}{\mathbf{F}}\right\}
\end{aligned}
$$

The spectral system matrix $[\mathbf{S}]$ for the complete intact structure system can be obtained by assembling all spectral element matrices as

$$
[\mathbf{S}(\omega)]=\sum_{k=1}^{N}\left[\mathbf{L}_{k}\right]^{T}\left[\mathbf{s}_{k}(\omega)\right]\left[\mathbf{L}_{k}\right]
$$

where $N$ is the number of finite elements and $\left[\mathbf{s}_{k}\right]$ is the spectral element matrix for the $k$-th finite element. The matrix $\left[\mathbf{L}_{k}\right]$ denotes the locator matrix, which locates the components of $\left[\mathbf{s}_{k}\right]$ into $[\mathbf{S}]$ for the assembly. If the spectral element matrix $\left[\mathbf{s}_{k}\right]$ is not available, it can be represented in terms of the conventional finite element matrices, as an approximation, as

$$
\left[\mathbf{s}_{k}\right]=\left[\mathbf{k}_{k}\right]-\omega^{2}\left[\mathbf{m}_{k}\right]
$$

where $\left[\mathbf{k}_{k}\right]$ and $\left[\mathbf{m}_{k}\right]$ are the finite element stiffness and mass matrices, respectively. In the similar sense, the perturbed spectral system matrix $[\Delta \mathbf{S}]$ can be obtained from

$$
[\Delta \mathbf{S}(\omega)]=\sum_{k=1}^{N}\left[\mathbf{L}_{k}\right]^{T}\left[\Delta \mathbf{s}_{k}(\omega)\right]\left[\mathbf{L}_{k}\right]
$$

where $\left[\Delta \mathbf{s}_{k}\right]$ is the perturbed spectral element matrix for the k-th element.

In the previous work [23], the presence of damage was represented by the degradation of Young's modulus as $\bar{E}=E(1-D)$, where $E$ and $\bar{E}$ are the Young's moduli for the intact and damaged states, respectively, and $D$ is damage magnitude, and showed that the perturbed spectral element matrix $\left[\Delta \mathbf{s}_{k}\right]$ can be approximated as

$$
\left[\Delta \mathbf{s}_{k}(\omega)\right] \cong D_{k}\left[\mathbf{s}_{k}(\omega=0)\right]=D_{k}\left[\mathbf{k}_{k}\right]
$$

where $D_{k}$ is 'effective' damage magnitude assumed to be uniform over the $k$-th finite element, and $\left[\mathbf{k}_{k}\right]$ is the conventional finite element stiffness matrix for the $\mathrm{k}$-th element.

By using Eqs (9) and (11), Eq. (7) can be rewritten in the form [25]

$$
[\Phi(\omega)]_{M \times N}\{\mathbf{D}\}_{N \times 1}=\{\mathbf{b}(\omega)\}_{M \times 1}
$$

where

$$
\begin{aligned}
& \{\mathbf{D}\}=\left\{\begin{array}{llll}
D_{1} & D_{2} & \ldots & D_{N}
\end{array}\right\}^{T} \\
& \{\mathbf{b}(\omega)\}=\{\boldsymbol{\delta}(\omega)\}+\frac{1}{\omega^{2}}[\mathbf{X}(\omega)]\left\{\mathbf{A}_{m}(\omega)\right\} \\
& {[\Phi(\omega)]=\left[\begin{array}{lllll}
\varphi(\omega) & \varphi_{2}(\omega) & \ldots & \varphi_{N}(\omega)
\end{array}\right]}
\end{aligned}
$$

with 


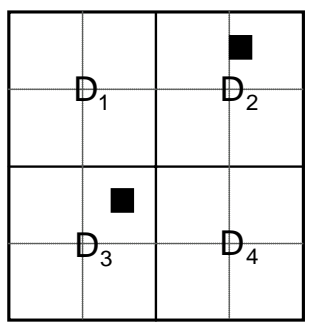

(a) The First Step: initial $2 \times 2$ finite elements

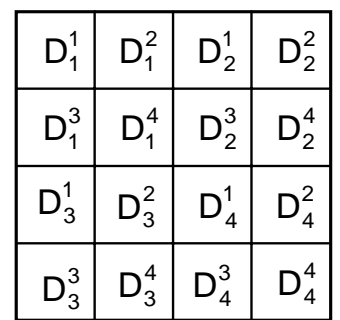

(b) The Second Step: $4 \times 4$ finer finite elements

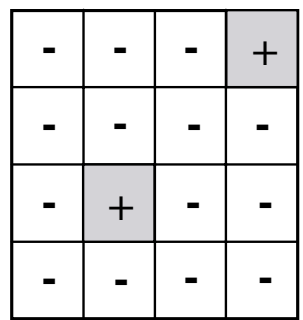

(c) The Third Step:

$(-)$ if $\mathrm{D}_{\mathrm{j}}^{\mathrm{i}}<\mathrm{D}_{\mathrm{j}}$

$(+)$ if $\mathrm{D}_{\mathrm{j}}^{1} \geq \mathrm{D}_{\mathrm{j}}$

Fig. 1. The conceptual illustration of three-steps process: in (a), $\mathbf{\square}$ indicates local damages.

$$
\begin{array}{r}
\left\{\boldsymbol{\varphi}_{k}(\omega)\right\}=-\frac{1}{\omega^{2}}\left(\left[\mathbf{L}_{k}\right][\mathbf{T}(\omega)]\right)^{T}\left[\mathbf{k}_{k}(\omega)\right]\left(\left[\mathbf{L}_{k}\right](15)\right. \\
[\mathbf{T}(\omega)])\left\{\mathbf{A}_{m}(\omega)\right\} \quad(k=1,2, \ldots, N)
\end{array}
$$

One may note from Eq. (13) that $[\Phi]$ and $\{\mathbf{b}\}$ are determined by only the spectral element matrix in the intact state $[\mathbf{S}]$, the nodal forces locator vector $\{\boldsymbol{\delta}\}$, and the measured inertance FRF $\left\{\mathbf{A}_{m}\right\}$. Solving Eq. (13) for $\{\mathbf{D}\}$ implies the structural damage identification.

Equation (13) represents $M$ linear algebraic equations (for a specific excitation frequency $\omega$ ) in $N$ unknown damage magnitudes $D_{k}$. Note $M$ is equal to the number of 'master' nodal DOF while $N$ is equal to the number of finite elements to be examined for unknown damage magnitudes $D_{k}$. In general $N>M$, because the number of measured nodal DOF is very limited in practice while a huge number of very fine finite elements should be used to search for unknown local damages. Thus, as a strategy to make the damage identification problem of Eq. (13) well-posed, one can properly vary the excitation frequency $\omega$ and collect more equations from Eq. (13) to form a ( $N$ by $N$ ) matrix equation as [25]

$$
[\Psi]_{N \times N}\{\mathbf{D}\}_{N \times 1}=\{\mathbf{B}\}_{N \times 1}
$$

where

$$
\begin{aligned}
& {[\Psi]_{N \times N}=} {\left[\begin{array}{c}
{\left[\Phi\left(\omega_{1}\right)\right]_{M \times N}} \\
{\left[\Phi\left(\omega_{2}\right)\right]_{M \times N}} \\
\vdots \\
{\left[\Phi\left(\omega_{r}\right)\right]_{M \times N}}
\end{array}\right], } \\
&\{\mathbf{B}\}_{N \times 1}=\left\{\begin{array}{c}
\left\{\mathbf{b}\left(\omega_{1}\right)\right\}_{M \times 1} \\
\left\{\mathbf{b}\left(\omega_{2}\right)\right\}_{M \times 1} \\
\vdots \\
\left\{\mathbf{b}\left(\omega_{r}\right)\right\}_{M \times 1}
\end{array}\right\} \\
&(N=r \times M)
\end{aligned}
$$

Note that Eq. (16) is formulated in a general form so that it can be applied to any structure in principle. However, in contrast to the 1-D or truss-like frame structures, it is quite complicated or difficult to formulate the spectral element matrix for the plate-like 2-D structures and 3D structures. Thus, for such complex structures, the spectral element matrix $[\mathbf{s}]$ required to set up Eq. (16) can be replaced with the approximate spectral element matrix $[\mathbf{s}]_{\text {approx }}=[\mathbf{k}]-\omega^{2}[\mathbf{m}]$, where $[\mathbf{k}]$ and $[\mathbf{m}]$ are the conventional finite element stiffness matrix and finite element mass matrix, respectively.

\subsection{Reduced-domain method}

If only $n$ finite elements among total $N$ finite elements are damaged, in other words, if $D_{n+1}=$ $D_{n+2}=\ldots=D_{N}=0$, the summation in Eq. (11) needs to be carried out only for the $n$ damaged finite elements as

$$
[\Delta \mathbf{S}(\omega)]=\sum_{k=1}^{n}\left[\mathbf{L}_{k}\right]^{T}\left[\Delta \mathbf{s}_{k}(\omega)\right]\left[\mathbf{L}_{k}\right](n<N)
$$

while the summation in Eq. (9) should be carried out for all $N$ finite elements. Accordingly the dimension of the matrix Eq. (16) can be reduced as follows:

$$
[\Psi]_{n \times n}\{\mathbf{D}\}_{n \times 1}=\{\mathbf{B}\}_{n \times 1}
$$

where

$$
\begin{aligned}
{[\Psi]_{n \times n} } & =\left[\begin{array}{c}
{\left[\Phi\left(\omega_{1}\right)\right]_{M \times n}} \\
{\left[\Phi\left(\omega_{2}\right)\right]_{M \times n}} \\
\vdots \\
{\left[\Phi\left(\omega_{r}\right)\right]_{M \times n}}
\end{array}\right], \\
\{\mathbf{B}\}_{n \times 1} & =\left\{\begin{array}{c}
\left.\mathbf{b}\left(\omega_{1}\right)\right\}_{M \times 1} \\
\left\{\mathbf{b}\left(\omega_{2}\right)\right\}_{M \times 1} \\
\vdots \\
\left\{\mathbf{b}\left(\omega_{r}\right)\right\}_{M \times 1}
\end{array}\right\}(n=r \times M)
\end{aligned}
$$


$\mathrm{D}=0.5$

\begin{tabular}{|l|l|l|l|l|l|l|l|}
\hline & & & & & & \\
\hline
\end{tabular}

\begin{tabular}{|c|c|c|c|c|c|c|c|c|c|c|}
\hline \multicolumn{2}{|c|}{$\begin{array}{c}\text { Random } \\
\text { Noise in FRF }\end{array}$} & \multicolumn{9}{|c|}{ Predicted Damage Magnitudes } \\
\hline \multirow{3}{*}{$0 \%$} & 1st Step & \multicolumn{3}{|c|}{$2.7 \mathrm{E}-2$} & \multicolumn{3}{|c|}{$7.7 \mathrm{E}-2$} & \multicolumn{3}{|c|}{ 2.7E-2 } \\
\hline & 2nd Step & $6.7 \mathrm{E}-4$ & $1.6 \mathrm{E}-3$ & $5.6 \mathrm{E}-3$ & $2.9 \mathrm{E}-2$ & $2.1 \mathrm{E}-1$ & $2.9 \mathrm{E}-2$ & $5.6 \mathrm{E}-3$ & $1.6 \mathrm{E}-3$ & $6.7 \mathrm{E}-4$ \\
\hline & 3rd Step & - & - & - & - & + & - & - & - & - \\
\hline \multirow{3}{*}{$1 \%$} & 1st Step & \multicolumn{3}{|c|}{$2.8 \mathrm{E}-2$} & \multicolumn{3}{|c|}{$7.8 \mathrm{E}-2$} & \multicolumn{3}{|c|}{$2.9 \mathrm{E}-2$} \\
\hline & 2nd Step & $4.2 \mathrm{E}-3$ & $3.4 \mathrm{E}-3$ & $7.4 \mathrm{E}-3$ & 3.0E-2 & $2.1 \mathrm{E}-1$ & $2.8 \mathrm{E}-2$ & $5.9 \mathrm{E}-3$ & $3.6 \mathrm{E}-3$ & $3.1 \mathrm{E}-3$ \\
\hline & 3rd Step & - & - & - & - & + & - & - & - & - \\
\hline \multirow{3}{*}{$3 \%$} & 1st Step & \multicolumn{3}{|c|}{$2.9 \mathrm{E}-2$} & \multicolumn{3}{|c|}{$7.9 \mathrm{E}-2$} & \multicolumn{3}{|c|}{$2.8 \mathrm{E}-2$} \\
\hline & 2nd Step & $1.5 \mathrm{E}-2$ & $9.6 \mathrm{E}-3$ & $9.8 \mathrm{E}-3$ & $2.6 \mathrm{E}-2$ & $2.1 \mathrm{E}-1$ & $3.2 \mathrm{E}-2$ & $1.8 \mathrm{E}-2$ & $9.4 \mathrm{E}-3$ & $1.2 \mathrm{E}-2$ \\
\hline & 3rd Step & - & - & - & - & + & - & - & - & - \\
\hline \multirow{3}{*}{$5 \%$} & 1st Step & \multicolumn{3}{|c|}{$2.9 \mathrm{E}-2$} & \multicolumn{3}{|c|}{$7.9 \mathrm{E}-2$} & \multicolumn{3}{|c|}{$3.1 \mathrm{E}-2$} \\
\hline & 2nd Step & $1.5 \mathrm{E}-2$ & $2.0 \mathrm{E}-2$ & $1.9 \mathrm{E}-2$ & $6.0 \mathrm{E}-2$ & $1.8 \mathrm{E}-1$ & $4.3 \mathrm{E}-2$ & $1.8 \mathrm{E}-2$ & $2.6 \mathrm{E}-2$ & $2.1 \mathrm{E}-2$ \\
\hline & 3rd Step & - & - & - & - & + & - & - & - & - \\
\hline \multirow{3}{*}{$7 \%$} & 1st Step & \multicolumn{3}{|c|}{$2.9 \mathrm{E}-2$} & \multicolumn{3}{|c|}{$8.0 \mathrm{E}-2$} & \multicolumn{3}{|c|}{$3.2 \mathrm{E}-2$} \\
\hline & 2nd Step & 1.6E-2 & $1.1 \mathrm{E}-2$ & $2.0 \mathrm{E}-2$ & $5.6 \mathrm{E}-2$ & $2.4 \mathrm{E}-1$ & $3.7 \mathrm{E}-2$ & $8.3 \mathrm{E}-3$ & $1.6 \mathrm{E}-2$ & $1.7 \mathrm{E}-2$ \\
\hline & 3rd Step & - & - & - & - & + & - & - & - & - \\
\hline \multirow{3}{*}{$10 \%$} & 1st Step & \multicolumn{3}{|c|}{$2.8 \mathrm{E}-2$} & \multicolumn{3}{|c|}{$8.4 \mathrm{E}-2$} & \multicolumn{3}{|c|}{$3.5 \mathrm{E}-2$} \\
\hline & 2nd Step & $3.4 \mathrm{E}-2$ & $1.8 \mathrm{E}-2$ & $2.9 \mathrm{E}-2$ & $3.7 \mathrm{E}-2$ & $2.1 \mathrm{E}-1$ & $1.4 \mathrm{E}-2$ & $1.5 \mathrm{E}-2$ & $1.2 \mathrm{E}-2$ & $3.8 \mathrm{E}-2$ \\
\hline & 3rd Step & + & - & + & - & + & - & - & - & + \\
\hline
\end{tabular}

Fig. 2. Numerical validation of the three-steps process with varying the level of the noise in measured FRF-data.

Equation (19) implies that, by removing damage-free parts of structure from the initial domain of problem, the size of the inverse problem of damage identification can be reduced drastically without degrading the accuracy of damage identification results at all. Thus, instead of examining whole domain of problem to search out damage (i.e., full-domain method; FDM), it is desirable first to reduce the domain of problem in advance by removing damage-free parts of structure and then to examine only the reduced domain of problem (i.e., reduced-domain method; RDM). To realize this idea of RDM, the locations and sizes of the damage-free parts of structure should be known in advance. However, unfortunately this is not true for most practical cases. Thus, a special methodology is required to search out surely damage-free parts of structure during the process of damage identification analysis.

In the previous study [25], it was numerically and experimentally shown that the damage magnitudes predicted in the finite elements containing damage inside become larger and larger, converging to the true damage values, as the total number of finite elements, $N$, is gradually increased by dividing the finite elements used in the previous analysis into the finer finite elements, while those predicted in the truly intact finite elements become smaller and smaller, converging to zero. The result seems to be natural as far as the structure model and the vibration data used for damage identification are sufficiently accurate and reliable. 


\section{$\mathrm{O}=$ Excitation point $; \quad X=$ Measurement points}

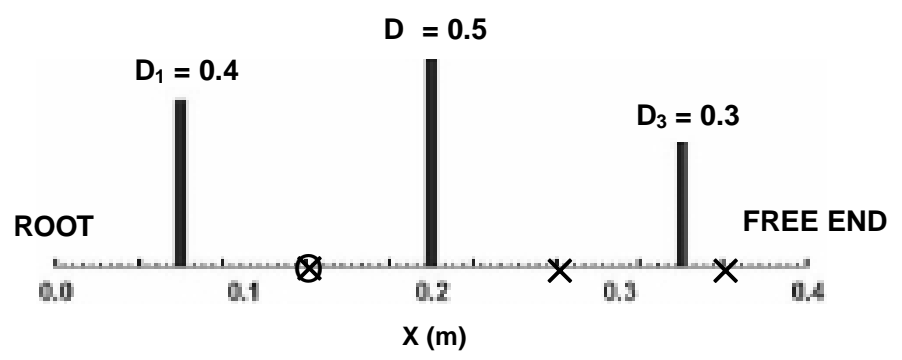

Fig. 3. Numerical example for the feasibility tests of the present reduced-domain method: a cantilevered beam with three local damages.

Motivated from the above observations, this study proposes a three-steps process to realize the idea of RDM, which is conceptually illustrated in Fig. 1.

1. The first step (Fig. 1a): Divide the domain of problem into $q$ finite elements and make the first damage prediction for the $q$ finite elements by using Eq. (16). The results are represented by $D_{j}(j=1,2, \ldots, q)$.

2. The second step (Fig. 1b): Divide each finite element used in the first step into $p$ finer subelements, which produces total $(p \times q)$ finite elements. Make the second damage prediction for the $(p \times q)$ finite elements. The results are now represented by $D_{j}^{i}(i=1,2, \ldots, p ; j=$ $1,2, \ldots, q)$.

3. The third step (Fig. 1c): If $D_{j}^{i}<D_{j}$, conclude that the ith sub-element within the jth finite element is damage-free. On the other hand, if $D_{j}^{i}>D_{j}$, conclude that the sub-element is possibly damaged. Based on this judgment, remove all damage-free sub-elements from the domain of problem by setting $D_{j}^{i}=0$ and then examine only the remained parts of domain for damage by using Eq. (19) in the next iteration, which starts again from the above first step.

By the iterative use of the present three-steps process of RDM, surely damage-free parts can be searched out and removed every iteration from the initial domain of problem to leave only damaged parts: this implies the location of damages. During the iterative process of RDM, the damage magnitudes can be quantified simultaneously from Eq. (19). As mentioned previously, however the present RDM becomes valid only when the above three-steps process can be successfully applied. Numerically simulated damage identification tests conducted in the present study show that the three-steps process works fairly well as far as the noises in FRFdata can be lowered below a certain level, which can be readily satisfied by most well-prepared vibration tests.

\section{Numerical and experimental tests}

\subsection{Numerical test of the three-steps process}

For successful application of the present RDM, the proposed three-steps process should work correctly. However, the validity of the three-steps process may depend on the accuracy of the structure model and the measured vibration data (i.e., FRF-data) used for damage identification. Thus, by assuming that the structure model used for damage identification is the refined structure model so that it is accurate enough, the validity of the three-step process will be tested numerically by varying the level of the noise in the measured FRFdata. As an illustrative example, a simply supported damaged beam is considered. As shown in the top of Fig. 2, the beam has the length $L=1.2 \mathrm{~m}$, bending stiffness $E I=11.2 \mathrm{~N}-\mathrm{m}^{2}$, and the mass density per length $\rho A=0.324 \mathrm{~kg} / \mathrm{m}$. A damage of effective magnitude $D=0.5$, uniformly distributed over the length $0.044 \mathrm{~m}$, is pre-specified at the center of the beam (see top of Fig. 2). The damage is then inversely identified by the full-domain method at the first two steps of the three-steps process. The example beam is modeled by the spectral Bernoulli-Euler beam element matrix given in Appendix. To represent the measurement noise in measured FRF-data, the random noise generated by the random noise generator function in MATLAB ${ }^{\circledR}$ is added to the theoretically computed FRF, as done by Thyagarajan et al. [23].

Figure 2 shows the damage identification results at the first two steps of the three-steps process, followed 


\section{1st Iteration}

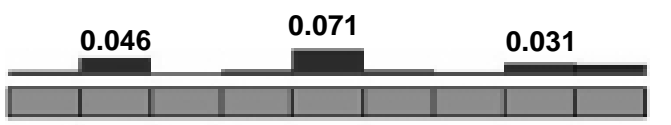

1st step

DIE = 2.06 e-01

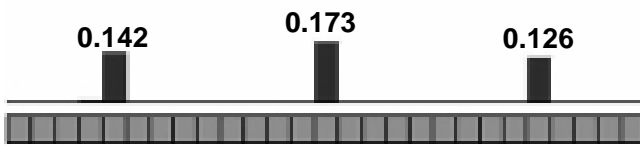

2nd step

DIE = 8.37 e-02

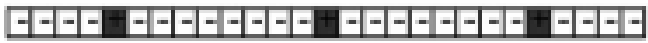

3rd step

\section{2nd Iteration}

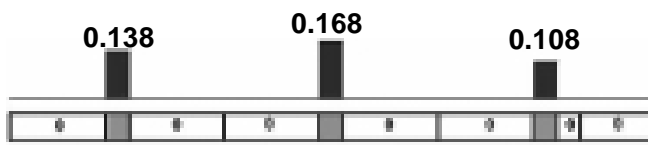

1st step

DIE = 8.94 e-02

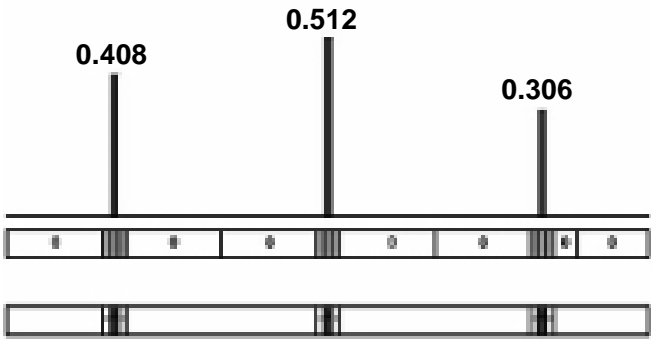

2nd step

DIE $=1.81$ e-03

\section{3rd Iteration}

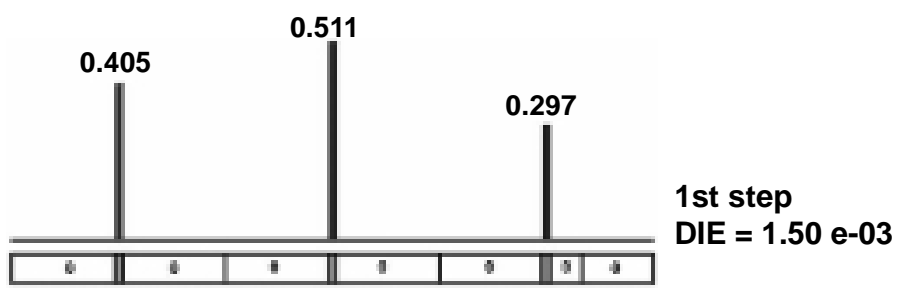

Fig. 4. Detailed procedure of the reduced-domain method: $0 \%$ random noise in FRF.

by the judgment at the last (i.e., third) step, obtained by varying the level of the noise in FRF-data. As shown in Fig. 2, the example beam is divided into total three finite elements at the first step. At the second step, the three finite elements used at the first step are then divided into three sub-elements to make total nine finite elements. When there exists no noise in the measured FRF-data (i.e., zero random noise), the damage magnitude in the middle finite element containing damage inside is predicted larger at the second step than at the first step, while the damage magnitudes in the other intact finite elements are predicted smaller at the second step than at the first step. Figure 2 shows that this is true up to about $9 \%$ noises in FRF-data. In other words, the three-steps process works correctly for the present example problem up to about $9 \%$ noises in measured FRF-data. For the successful applications of the present three-steps-process-based RDM, the noise in the true measured FRF-data should be minimized as low as possible, probably less than about 5\%, which can be met by most well-prepared vibration tests. As an additional effort to improve the reliability of the present three-steps process, one may repeat the first two steps several times by using further finer finite elements. 


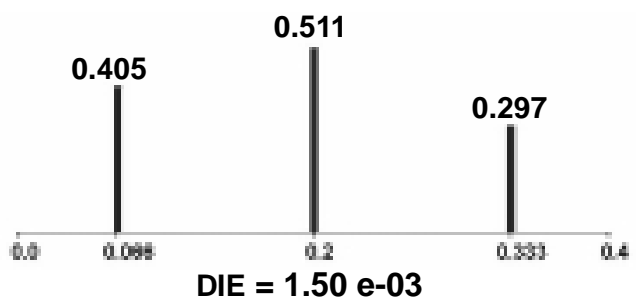

(a) $0 \%$ Random Noise

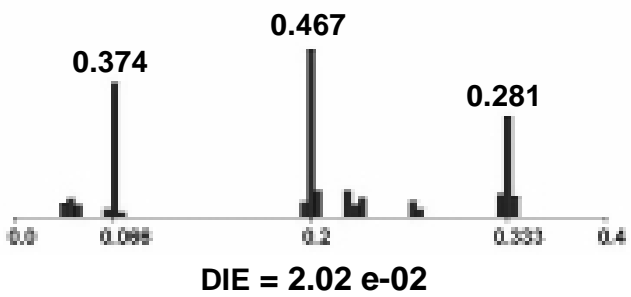

(c) $5 \%$ random noise

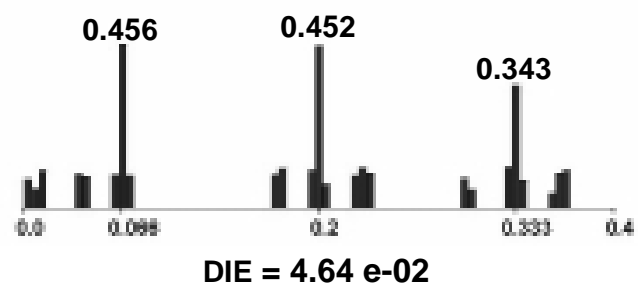

(e) $10 \%$ Random Noise

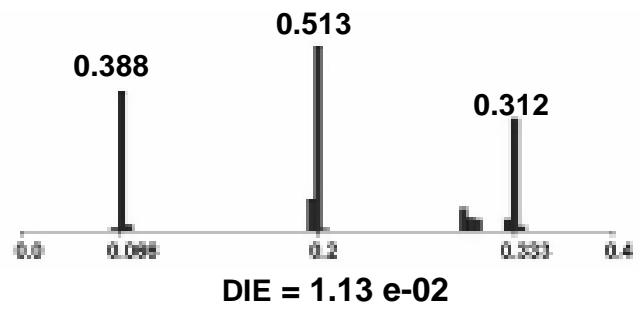

(b) $3 \%$ random noise

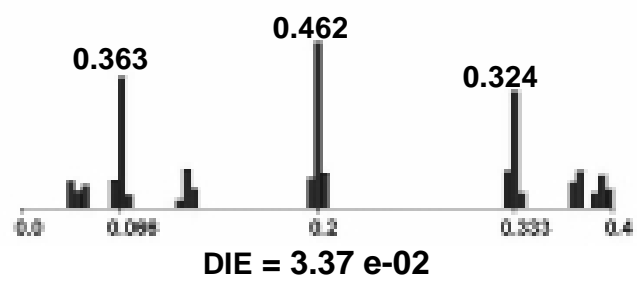

(d) $7 \%$ random noise

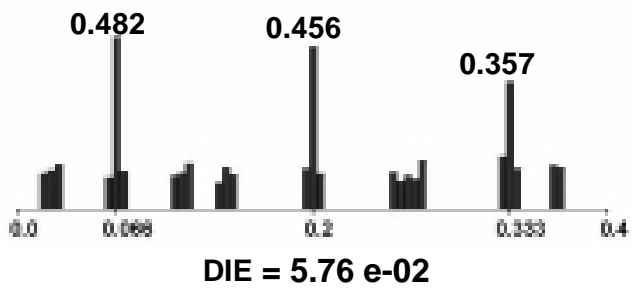

(f) $12 \%$ Random Noise

Fig. 5. The effects of the noise in measured FRF-data on the damage identification results obtained by the reduced-domain method.

\subsection{Numerical test of the reduced-domain method}

The numerical feasibility tests for the present RDM are conducted for a cantilevered Euler-Bernoulli beam. As shown in Fig. 3, the beam has the length $L=0.4 \mathrm{~m}$, bending stiffness $E I=14.6 \mathrm{~N}-\mathrm{m}^{2}$, and the mass density per length $\rho A=0.275 \mathrm{~kg} / \mathrm{m}$. The spectral Bernoulli-Euler beam element model given in Appendix is used. For the feasibility tests, three local damages are pre-specified along the beam, as shown in Fig. 3, and then inversely identified by applying the present RDM. The three local damages have the magnitudes $D_{1}=0.4, D_{2}=0.5$, and $D_{3}=0.3$. In Fig. 3, the circle $(\bigcirc)$ indicates the location of the applied harmonic point force (i.e., $x=0.133 \mathrm{~m}$ ) and the crosses $(\times)$ indicate the FRF measurement points (i.e., $x=0.133,0.267$, and $0.356 \mathrm{~m}$ ).

Figure 4 is given to show the detailed procedure of the present RDM, in which the three-steps process is applied every iteration, without considering the noise in the measured FRF-data. In the first iteration, as shown in Fig. 4, the beam is divided into nine (equal) finite elements at the first step, and then into 27 finite elements at the second step. Because the FRF-data are measured at three points (see Fig. 3), three excitation frequencies are used at the first step to obtain total nine equations for the nine finite elements model, while nine excitation frequencies are used at the second step to obtain total 27 equations for the 27 finite elements model. The excitation frequencies are chosen in the low frequency range for the better damage identification, based on the discussion in the previous work [25]. In the third step, by comparing the damage magnitudes predicted at the first and second steps, the sub-elements in which the predicted damage becomes larger at the second step are indicated by the plus $(+)$ sign, whereas the other sub-elements are indicated by the minus $(-)$ 


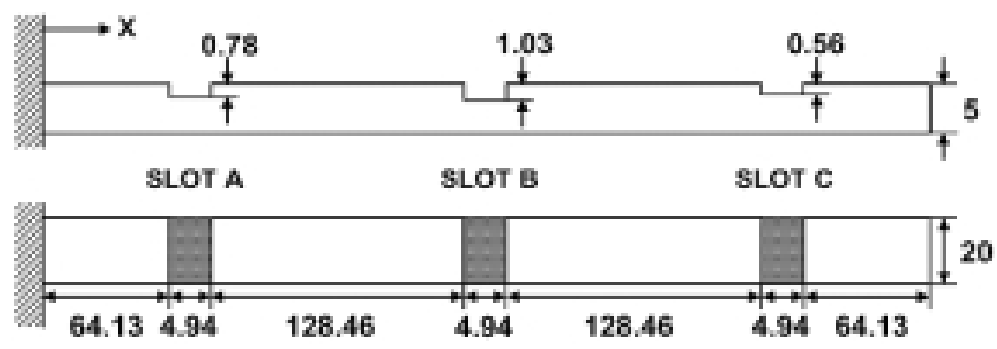

Fig. 6. Geometry of a damaged beam specimen (units: $\mathrm{mm}$ ).

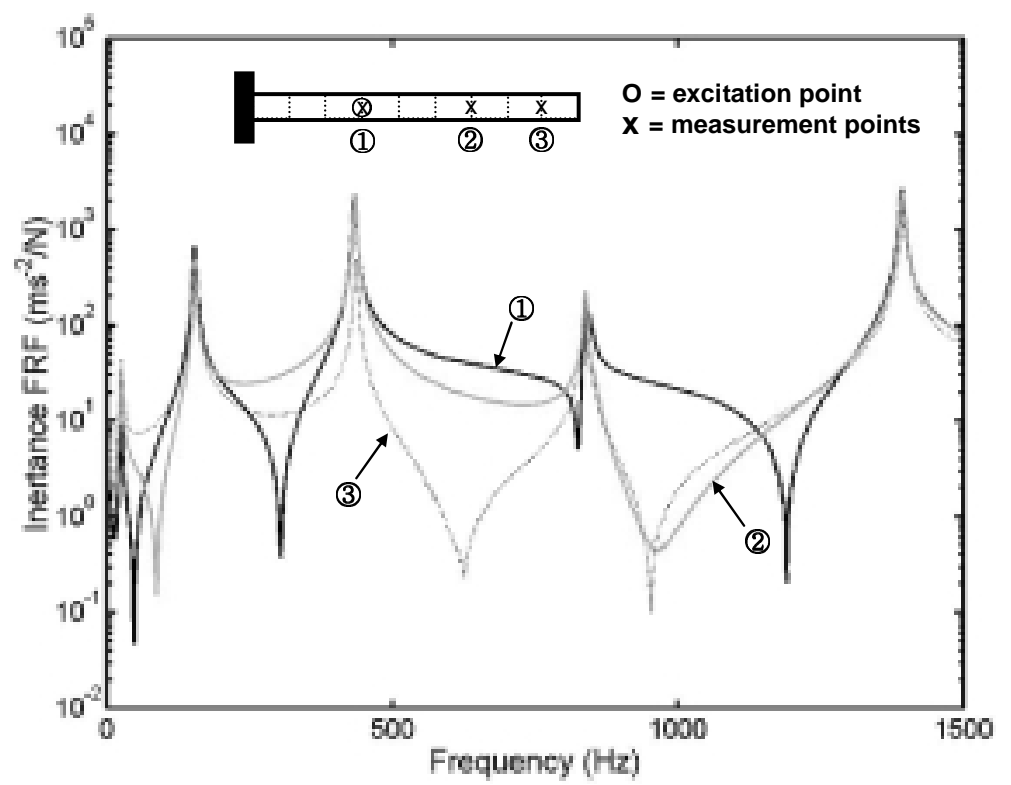

Fig. 7. The inertance FRF curves measured at $x=0.133 \mathrm{~m}$ (point 1), $0.267 \mathrm{~m}$ (point 2), and $0.356 \mathrm{~m}$ (point 3 ) of the damaged beam.

sign. In this study, as shown in Fig. 4, the accuracy of the predicted damage distribution along the beam with respect to the true damage distribution is represented by the damage identification error (DIE) defined by

$$
\mathrm{DIE} \equiv \sqrt{\frac{1}{L} \sum_{j}^{N} l_{j}\left(D_{j}^{\text {Pred }}-D_{j}^{\text {True }}\right)^{2}}
$$

where $L$ is the total length of beam and $D_{j}$ is the damage magnitude in the $j$ th finite element of length $l_{j}$. The superscripts "True" and "Pred" denote the true and predicted damages, respectively. The smaller DIE, the more accurate damage identification.

Figure 4 shows that, in the first iteration, the damage magnitudes in the truly damaged finite elements are indeed predicted larger at the second step, whereas those in the truly intact finite elements are predicted smaller. Thus, in the third step, only the 5th, 14th, and 23rd finite elements are marked by the plus sign, whereas the other finite elements are marked by the minus sign. In the first step of the second iteration, the finite elements marked by the minus signs are all removed from the domain of problem by setting $D=0$, and only the remaining three finite elements marked by the plus signs are examined for damage. In the next step, the three finite elements are divided into three finer sub-elements to make total nine finite elements. By comparing the damage prediction for the three finite elements at the first step with that for the nine sub-elements at the second step, one may see that the damage is predicted larger only in the three centered sub-elements marked by the plus sign. Figure 4 clearly shows that, if no noise exists in the measured FRF-data, the present RDM can locate and quantify all local damages very accurate.

Figure 5 shows the effects of the noise in measured FRF-data on the accuracy of the damage identification results obtained by the present RDM. The results shown in Fig. 5 are obtained from the mean values of five sim- 


\section{1st Iteration}
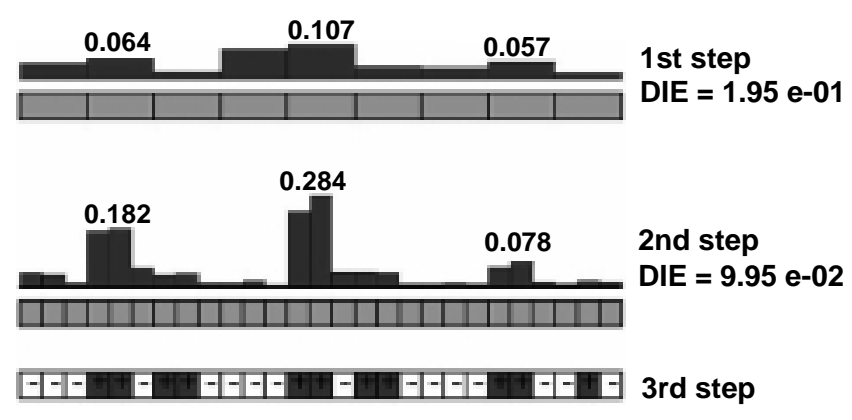

\section{2nd Iteration}
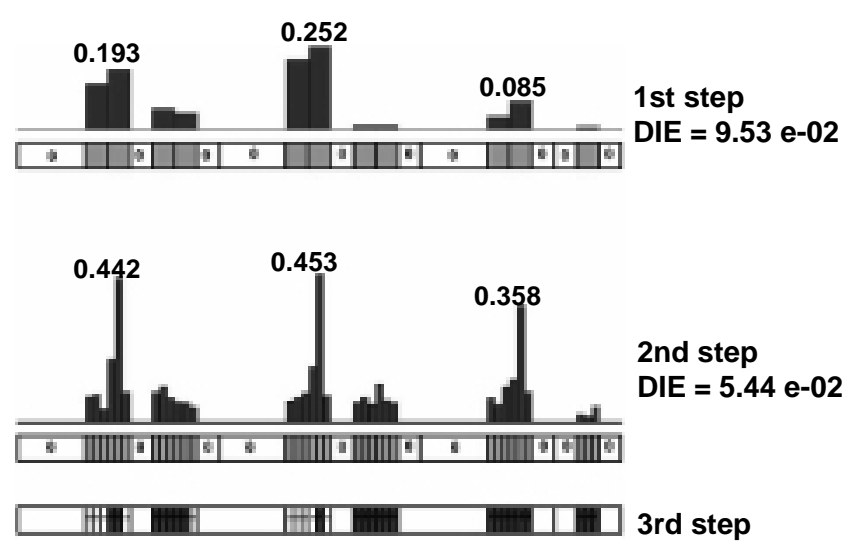

\section{3rd Iteration}

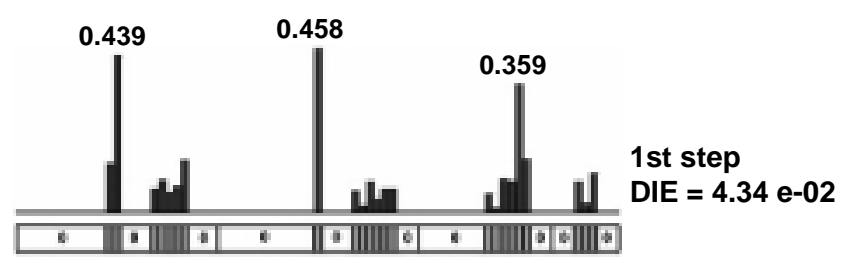

Fig. 8. Experimentally measured FRF-data based damage identification results obtained by the reduced-domain method.

ulations conducted for each level of noise in FRF-data, which is increased up to $12 \%$. As expected, increasing the level of noise in FRF-data indeed degrades the accuracy of damage identification. For the present example problem, the RDM is found to fairly well locate and quantify all pre-specified local damages up to about $9 \%$ noise in FRF-data.

\subsection{Experimental tests}

Figure 6 shows a cantilevered beam specimen used for experiments. The beam is $0.4 \mathrm{~m}$ long, $0.02 \mathrm{~m}$ wide, and $0.005 \mathrm{~m}$ thick. The beam has the mass density $\rho A=0.275 \mathrm{~kg} / \mathrm{m}$ and the bending stiffness $E I=14.6 \mathrm{~N}-\mathrm{m}^{2}$, all determined by experiments. The intact beam is damaged by introducing three slots of $4.94 \mathrm{~mm}$ wide and $0.78,1.03$, and $0.56 \mathrm{~mm}$ deep: i.e., the slot A, slot B and slot $C$ in Fig. 6. The depths of the slot $\mathrm{A}$, slot $\mathrm{B}$, and slot $\mathrm{C}$ are so determined that the corresponding effective damages become $0.4,0.5$, and 0.3 , respectively. Thus, the damaged beam specimen may represent the numerical example problem considered in the previous section (see Fig. 3). To excite the damaged beam specimen, an impulse point force is applied at $x=0.133 \mathrm{~m}$ (point 1 ) by the shaker B\&K 4810. As shown in Fig. 7, the (inertance) FRF 
curves are measured at three points $x=0.133 \mathrm{~m}$ (point 1), $0.267 \mathrm{~m}$ (point 2), and $0.356 \mathrm{~m}$ (point 3 ) by using the accelerometer B\&K 4374 and signal analyzer HP 35670A. The FRF mesurement is repeated many times until the FRF-data showing a very satisfactory coherence [32] is measured. The result is shown in Fig. 7.

Figure 8 shows the damage identification results obtained by the present RDM, using the experimentally measured FRF-data shown in Fig. 7. As well proved by the previous numerical feasibility tests, the present results also show that the present RDM can fairly well locate and quantify all damages, i.e., the three slots. The discrepancies between the predicted damage distribution and the true damage distribution may be mostly due to the noise in measured FRF-data and the error in the structure model. Comparing Figs 5 and 8, one may observe that the damage distribution predicted by using the real measured FRF-data is quite similar to that predicted by using the analytically simulated FRF-data, considering about $7 \%$ to $9 \%$ random noises.

\section{Conclusions}

In the present study, a reduced-domain method is applied to a SEM-based structural damage identification theory developed in the author's previous work, and then its feasibility is tested experimentally as well as numerically. In the reduced-domain method, a threesteps process is used to reduce the domain of problem by iteratively searching out and removing damage-free parts of structure in the course of the damage identification analysis. Numerical tests show that, the reduceddomain method works quite well, for the example problem, up to about 9\% noises in FRF-data. Experimental tests conducted for a damaged cantilevered beam also shows that the reduced-domain method fairly well locates and quantifies all local damages (i.e., three slots). However, for the successful application of the present reduced-domain method to the practical problems, the level of the noise in measured FRF-data should be lowered below a certain value, in general, depending on the size and strength of the damage.

\section{References}

[1] W.J. Thompson, Vibration of slender bars with discontinuities in stiffness, Journal of Applied Mechanics 17 (1943), 203207.
[2] P.G. Kirmsher, The effect of discontinuities on the natural frequencies of beams, Proceedings of American Society of Testing and Materials 44 (1944), 897-904.

[3] A.D. Dimarogonas, Vibration of cracked structures: a state of the art review, Engineering Fracture Mechanics 55 (1996), $831-857$.

[4] S.W. Doebling, C.R. Farrar, M.B. Prime and D.W. Shevitz, Damage identification and health monitoring of structural and mechanical systems from changes in their vibration characteristics: a literature review, Los Alamos National Laboratory Reports LA-13070-MS, UC-900, 1996.

[5] O.S. Salawu, Detection of structural damage through changes in frequency: a review, Engineering Structures 19 (1997), $718-723$.

[6] Y. Zou, L. Tong and G.P. Steven, Vibration-based modeldependent damage(delamination) identification and health monitoring for composites structures - a review, Journal of Sound and Vibration 230 (2000), 357-378.

[7] J.D. Collins, G.C. Hart, T.K. Hasselman and B. Kennedy, Statistical identification of structures, AIAA Journal 12 (1974), 185-190.

[8] A.M. Kabe, Stiffness matrix adjustment using mode data, AIAA Journal 28 (1985), 1431-1436.

[9] D.C. Zimmermann and M. Kaouk, Structural damage detection using a minimum rank update theory, Journal of Vibration and Acoustics 116 (1994), 222-231.

[10] D. Armon, Y. Ben-Haim and S. Braun, Crack detection in beams by rank-ordering of eigenfrequency shifts, Mechanical Systems and Signal Processing 8 (1994), 81-91.

[11] G. Hearn and R.B. Testa, Modal analysis for damager detection in structures, Journal of Engineering Mechanics 117 (1991), 3042-3063.

[12] A. Morassi, Crack-induced changes in eigenparameters of beam structures, Journal of Mechanical Engineering 119(9) (1993), 1798-1803.

[13] N. Bicanic and H.P. Chen, Damage identification in framed structures using natural frequencies, International Journal of Numerical Methods in Engineering 40 (1997), 4451-4468.

[14] S. Hassiotis, Identification of damage using natural frequencies and Markov parameters, Computers and Structures $\mathbf{7 4}$ (2000), 365-373.

[15] A.K. Pandey, M. Biswas and M.M. Samman, Damage detection from changes in curvature mode shapes, Journal of Sound and Vibration 145 (1991), 321-332.

[16] M.M. Abdel Wahab and G. De Roeck, Damage detection in bridges using modal curvatures: application to a real damage scenario, Journal of Sound and Vibration 226 (1999), 217235.

[17] J.S. Lew, Using transfer function parameter changes for damage detection of structures, AIAA Journal 33 (1995), 21892193.

[18] A.K. Pandey and M. Biswas, Damage diagnosis of truss structures by estimation of flexibility change, International Journal of Analytical and Experimental Modal Analysis 10 (1995), 104-117.

[19] J.M. Ricles and J.B. Kosmatka, Damage detection in elastic structures using vibratory residual forces and weighted sensitivity, AIAA Journal 30 (1992), 2310-2316.

[20] I. Sheinman, Damage detection and updating of stiffness and mass matrices using mode data, Computers and Structures 59 (1996), 149-156.

[21] K.C. Park, G.W. Reich and K.F. Alvin, Structural damage detection using localized flexibilities, Journal of Intelligent Material Systems and Structures 9 (1998), 911-919. 
[22] Z. Wang, R.M. Lin and M.K. Lim, Structural damage detection using measured FRF data, Computer Methods in Applied Mechanics and Engineering 147 (1997), 187-197.

[23] S.K. Thyagarajan, M.J. Schulz and P.F. Pai, Detecting structural damage using frequency response functions, Journal of Sound and Vibration 210 (1998), 162-170.

[24] G. Park, H.H. Cudney and D.J. Inman, An integrated health monitoring technique using structural impedance sensors, Journal of Intelligent Material Systems and Structures $\mathbf{1 1}$ (2000), 448-455.

[25] U. Lee and J. Shin, A frequency-domain method of structural damage identification formulated from dynamic stiffness equation of motion, Journal of Sound and Vibration 257(4) (2002), 615-634.

[26] J.F. Doyle, Wave Propagation in Structures: Spectral Analysis Using Fast Discrete Fourier Transforms, Springer-Verlag, New York, 1997.

[27] U. Lee, J. Kim and A.Y.T. Leung, The spectral element method in structural dynamics, The Shock and Vibration Digest 32 (2000), 451-465

[28] P.E. McGowan, Dynamic test/ analysis correlation using reduced analytical models, M.S. Thesis, Old Dominion University, 1992.

[29] T. Simmermacher, Damage detection and model refinement of coupled structural systems, Ph.D. Thesis, University of Houston, 1996.

[30] G.H. James and D.C. Zimmerman, Reduction/expansion studies for damage identification of continuous aerospace structures, Proceedings of International Modal Analysis Conference, 1997, 1772-1778.

[31] T. Marwala and P.S. Heyns, Multiple-criterion method for determining structural damage, AIAA Journal 36 (1998), 1494 1501.
[32] D.J. Ewins, Modal Testing: Theory and Practice, Research Studies Press Ltd, Hertfordshire, 1984.

\section{Appendix}

The spectral element matrix for an intact finite Bernoulli-Euler beam element is given by $[26,27]$

$$
[\mathbf{s}(\omega)]=\frac{\kappa E I}{C h \cdot c-1}\left[\begin{array}{ll}
\mathbf{s}_{1} & \mathbf{s}_{2} \\
\mathbf{s}_{2}^{T} & \mathbf{s}_{3}
\end{array}\right]
$$

where

$$
\begin{aligned}
& {\left[\mathbf{s}_{1}\right]=\left[\begin{array}{lr}
-\kappa^{2}(C h \cdot s+S h \cdot c) & -\kappa \cdot S h \cdot s \\
-\kappa \cdot S h \cdot s & -(C h \cdot s-S h \cdot c)
\end{array}\right]} \\
& {\left[\mathbf{s}_{2}\right]=\left[\begin{array}{lr}
\kappa^{2}(S h+s)-\kappa(C h-c) \\
\kappa(C h-c)-(S h-s)
\end{array}\right]} \\
& {\left[\mathbf{s}_{2}\right]=\left[\begin{array}{cr}
-\kappa^{2}(C h \cdot s+S h \cdot c) & \kappa \cdot S h \cdot s \\
-\kappa \cdot S h \cdot s & -(C h \cdot s-S h \cdot c)
\end{array}\right]}
\end{aligned}
$$

with

$$
\begin{aligned}
& s=\sin \kappa l, c=\cos \kappa l, S h=\sinh \kappa l, \\
& C h=\cosh \kappa l
\end{aligned}
$$



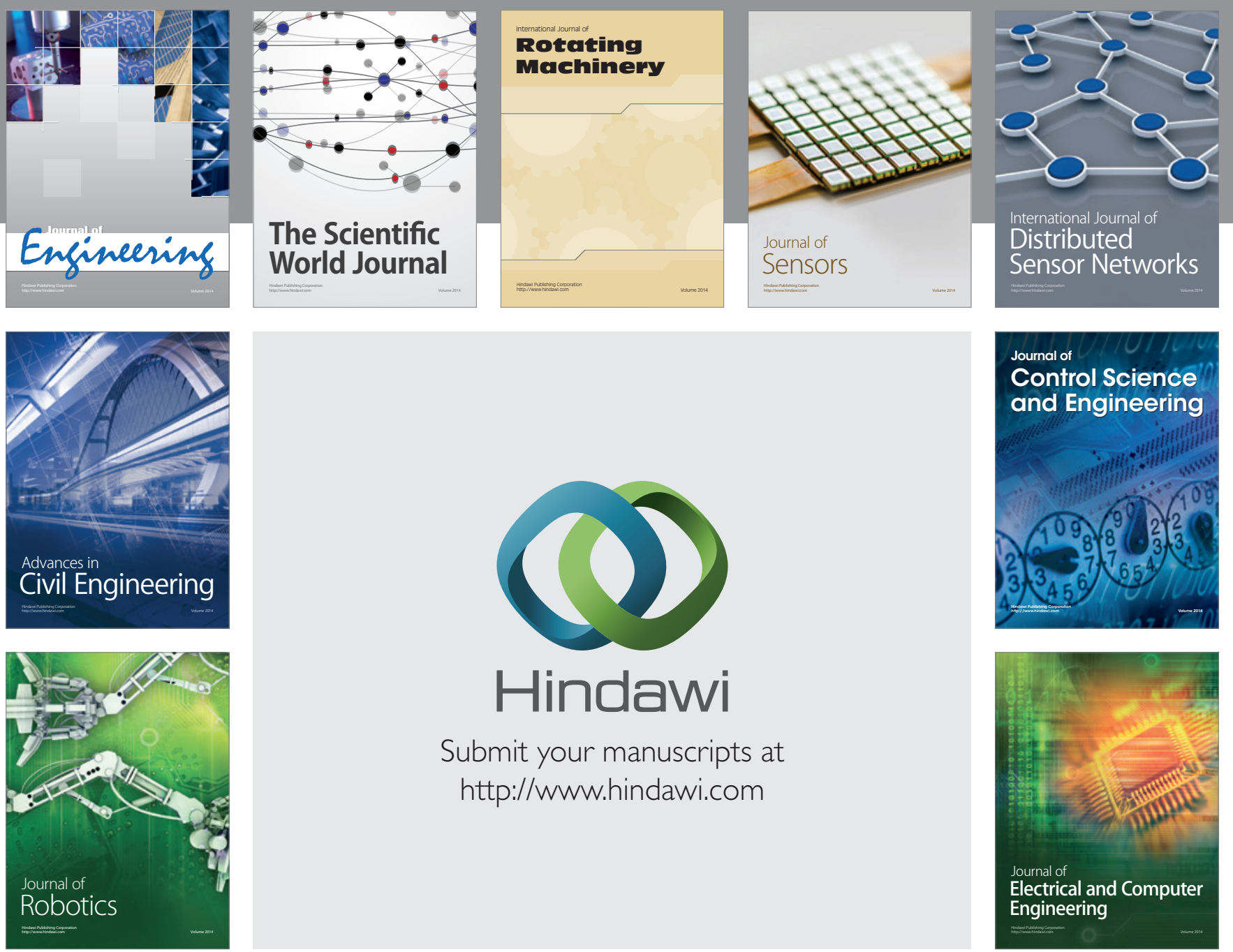

Submit your manuscripts at

http://www.hindawi.com
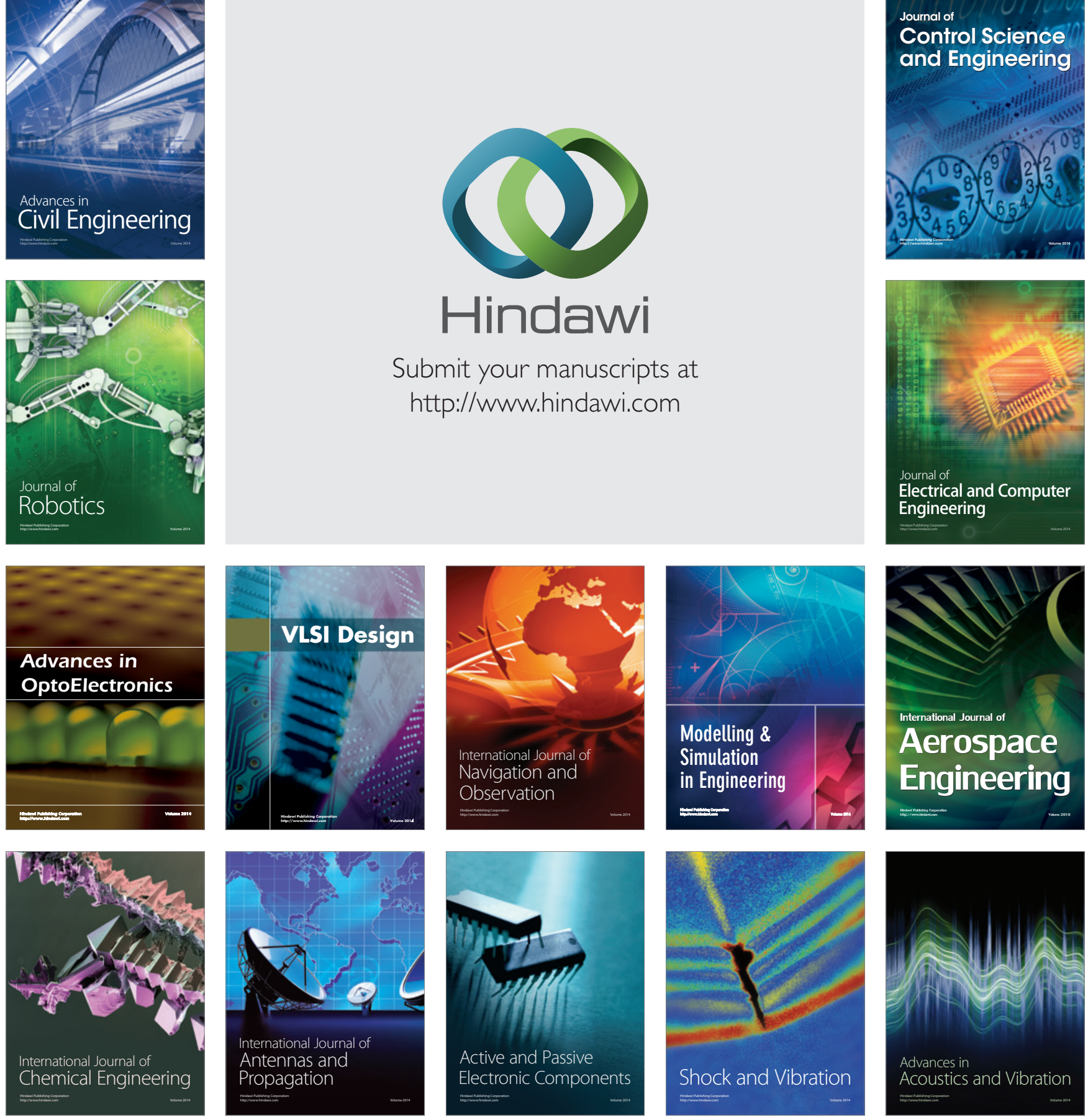